\title{
A REMARK ON THE COMMUTATIVITY OF ALGEBRAIC RINGS
}

\author{
TADASI NAKAYAMA
}

Let $F$ be a commutative ring with unit element 1 . A ring $R$ is called an algebraic ring over $F$ by Drazin, in his recent paper [2], when $R$ is an algebra ${ }^{11}$ over $F$ and every element $x$ of $R$ satisfies an equation of lower monic form

$$
x^{m}+\alpha_{m+1} x^{m+1}+\ldots+\alpha_{n} x^{n}=0
$$

with coefficients $\alpha_{i}$ in $F$. With this terminology and in generalization of some results of Arens-Kaplansky [1], Herstein [5], Jacobson [6], he proves in the same paper:

Let $F$ be either i) the ring of rational integers, or ii) a field of prime characteristic algebraic over its prime field, or iii) an algebraically closed field. Then, if $R$ is an algebraic ring over $F$ and if every nilpotent element in $R$ is central, $R$ is commutative.

Now, a common feature in i) and ii) is that $F$ is generated by a single element. Indeed, by making use of the writer's [9] generalization of Herstein's [5] theorem we may prove that the above statement holds whenever $F$ is (ring-) generated by a finite number of elements.") Also the case iii) can correspondingly be generalized to the case of a (commutative) ring which is (ring-)generated by an algebraically closed subfield $K$ and a finite (possibly void) set of elements. A further generalization is obtained by replacing here the algebraically closed subfield $K$ with a subring $K$ such that an integrity domain which is a homomorphic image of $K$ is always an algebraically closed field. We may also regard the case with a finite set of generators as having

Received July 18, 1958.

1) Thus $R$ admits $F$ both as a ring of left operators and a ring of right operators and the equations $\alpha x=x x, \alpha(x y)=(x x) y,(x y) x=x(y x)$ hold for all $x, y \in R$ and $\alpha \in F$. Together with Drazin [2] we explicitly remark that a non-zero element of $F$ may induce 0-endomorphism on $R$.

2) A direct application of [9], Satz 2 is allowed provided $R$ possesses a unit element. The case of $R$ having no unit element can, however, be settled by an easy modification of the proof there; cf. below too. 
$K=0$. Thus,

THEOREM. Let F be a commutative ring with unit element which is (ring-) generated by a finite number of elements and a subring $K$ (which may be 0) such that for every prime ideal $p$ of $K$ the residue ring $K / p$ is a field and algebraically closed." ') Then, if $R$ is an algebraic ring over $F$ and if every nilpotent element in $R$ is central, $R$ is commutative.

Proof. We quote from Drazin's paper [2] (cf. the converse part of Theorem 5.2 there):

Lemma. Let $R$ be a ring such that every nilpotent element in $R$ is central, i.e. lies in the center $Z$ of $R$. If $a^{t}=a^{t} b a^{t}$ with some elements $a, b$ in $R$ and a natural number $t$, then $a-a^{t+1} b \in Z$.

If $R$ is an algebraic ring over a commutative ring $F(\exists 1)$, then for every $a \in R$ there are clearly a polynomial $b$ in $a$ over $F$ and a natural number $t$ satisfying $a^{t}=a^{2 t} b\left(=a^{t} b a^{t}\right)$. If every nilpotent element of $R$ is central, then by the above lemma, $a-a^{t+1} b=a-a^{2} b_{1} \in Z$ with $b_{1}=a^{t-1} b$ or $b$ according as $t>$ or $=1$. If here $F$ is finitely (ring-)generated and $R$ has a unit element, then $b_{1}$ is contained in the subring of $R$ generated by $a$ and a certain finite number of elements of $Z$ independent of $a$. It follows then from [9], Satz 2 that $R$ is commutative. However, in order to consider the case of $R$ having no unit element and also to consider the case of $F$, as described in our Theorem, with $K \neq 0$, we want to repeat, with some modification, a part of our proof in [9], which is in turn a generalization and a refinement of Herstein's proof in [5].

Thus, we begin with assuming that $R$ is an algebraic ring over a commutative ring $F(\ni 1)$ and that every nilpotent element in $R$ is central. As we

3) $K=0$ satisfies this condition. An instance of $F$ with $K=0$, thus a commutative ring ( $\exists 1$ ) with a finite set of generators is provided by any ring ( $\exists 1)$ of (not necessarily all) integers in an algebraic number field of finite degree. More generally, let $L$ be a field (field-) generated by a finite number of elements and let $K_{1}, \ldots, K_{k}$ be any finite set of elements in $L$. Let $K$ be a ring consisting of (not necessarily all) elements in $L$ integral with respect to the ring $\left[K_{i}\right]$ (ring-) generated by $K_{1}, \ldots, K_{k}$ and containing $\left[K_{i}\right]$.. Then $K$ is (ring-)generated by a finite number of elements.

4) An instance of $K$ is the ring of formal power series in one variable over an algebraically closed field. We prefer this example to a polynomial ring in one variable over an algebraically closed field, since the latter would simply lead to an $F$ which is generated by a finite set of elements and an algebraically closed field. 
have observed above, for every $a \in R$ there is a polynomial $b$ in $a$ over $F$ such that $a-a^{2} b \in Z$. So, $R$ is in particular a $\xi$-ring in the sense of Drazin [3] and Utumi [10].

Now, assume that $R$ is subdirectly irreducible, and assume firstly that $R$ is semisimple (in Jacobson's sense). Then ([2], Lemma 4.1; cf. also [5], No. 2 ; [9], Hilfssatz 3) $R$ is necessarily a division ring. Assume further that $F$ has the structure of our Theorem, that is, that $F$ is generated by a finite set of elements $\alpha_{1}, \ldots, \alpha_{r}$ and a subring $K$ such that any integrity domain which is a homomorphic image of $K$ is necessarily an algebraically closed field. Let 1 be the unit element of (the division ring) $R$. The subring $1 F$ of $R$ is generated by $1 \alpha_{1}, \ldots, 1 \alpha_{r}$ and $1 K$. Here $1 K$ is either 0 or an algebraically closed field. If $1 K=0$, then Satz 1 in [9], or even its weaker formulation in the introduction there, suffices to secure the commutativity of $R$. So, consider the case $1 K \neq 0$. Assume that $R$ is non-commutative, i.e. that $R$ does not coincide with its center $Z$. By a theorem of Noether, Köthe, Albert and Jacobson ([6], Lemma 2) $R$ contains a finite separable proper extension $L$ of $Z$. Since $1 K(\leqq 1 F \leqq Z)$ is algebraically closed, this implies that $Z \neq 1 K$ and indeed $Z$ is transcendental over $1 K$. A portion of our proof to Hilfssatz 1 in [9] (cf. also Nagata-Nakayama-Tsuzuku [8]) suffices then to show that there is an (exponential) valuation $\rho$ of $L$ such that $\rho\left(1 \alpha_{\nu}\right) \geqslant 0, \nu=1, \ldots, r$, and which is constant on $1 K^{j)}$ and unramified of degree 1 over $Z$. We have $\rho(b) \geqslant 0$ for every $b \leqq 1 F$. Let $\rho^{\prime}$ be a valuation (which exists) different from $\rho$ and coincideing with $\rho$ on $Z$. Let $c, c^{\prime}$ be elements of $L$ such that $\rho(c)>0, \rho^{\prime}(c)=0, \rho\left(c^{\prime}\right)=0, \rho^{\prime}\left(c^{\prime}\right)>0$. Let $k$ be a natural number such that $k \rho(c)>\rho^{\prime}\left(c^{\prime}\right)$ and put $a=c^{k} c^{\prime}$. Then $\rho(a)=$ $k \rho(c)>0, \rho^{\prime}(a)=\rho^{\prime}\left(c^{\prime}\right)>0$, and $\rho(a)>\rho^{\prime}(a)$. Hence $a \notin Z$. Since $\rho\left(a^{2} b\right)>\rho(a)$, for $b \in 1 F$, we have $\rho\left(a-a^{2} b\right)=\rho\left(a^{2} b\right)=2 \rho(a)+\rho(b) \geqq 2 k \rho(c)+\rho(b)>2 \rho^{\prime}\left(c^{\prime}\right)+$ $\rho(b)$, while $\rho^{\prime}\left(a-a^{2} b\right)=\rho^{\prime}\left(a^{2} b\right)=2 \rho^{\prime}\left(c^{\prime}\right)+\rho^{\prime}(b)=2 \rho^{\prime}\left(c^{\prime}\right)+\rho(b)$. Hence $a-a^{2} b$ can not belong to $Z$, and this contradiction proves $R=Z$.

The case of a semisimple subdirectly irreducible $R$ of our theorem being thus settled, we turn to the case of a non-semisimple subdirectly irreducible $R$. We assume that it is an algebraic ring over a commutative ring $F(\ni 1$ ) (first without assuming the condition on the generation of $F$ ) and that its nilpotent elements are all central. Let $S$ be the unique smallest non-zero ideal of $R$,

5) On the set of non-zere elements of $1 K$, to be more precise. 
and set

$$
t(S)=\{a \in R \mid a S=S a=0\}
$$

From the fact that for every $a \in R$ there is $b \in R$ commutative with $a$ and satisfying $a-a^{2} b \in Z$, it follows (Herstein [5], p. 867; Nakayama [9], Hilfssatz 4) that $S \subseteq Z, R / t(S)$ is commutative, and $S$ is contained in every non-zero left, or right, ideal of $R$. If $t(S) \neq R$, then (Drazin [3], Theorem 7) $R / t(S)$ is a field.

On using the fact that for each $a \in R$ the element $b$ as above may be chosen to be a polynomial in $a$ over $F$, we may show, by a slight modification of an argument of Herstein [5], Theorem 11 (cf. [9], Hilfssatz 5), that $t(S$ ) is contained in $Z$.

Our aim is to prove $R=Z$. Since we have seen $t(S) \leqq Z$, we have only to consider the case $t(S) \neq R$; then $R / t(S)$ is a field as has been observed. Assume $R \neq Z$, contrary to our assertion, and let $a$ be an element of $R$ which

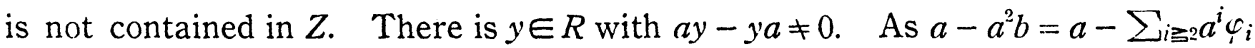
$\in Z$, with $\varphi_{i} \in F$, we have

$$
\left(\sum_{i \geqq 2} a^{i} \varphi_{i}\right) y-y\left(\sum_{i \geqq 2} a^{i} \varphi_{i}\right)=a y-y a .
$$

As here $a y-y a \in t(S) \leqq Z$ we verify readily that the left-hand side of the equality is equal to $\left(\sum_{i \geqq 2} i a^{i-1} \varphi_{i}\right)(a y-y a)$. Hence the equality implies

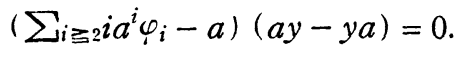

Since the righ-ideal of $R$ generated by $a y-y a(\neq 0) i s \neq 0$, whence contains $S$

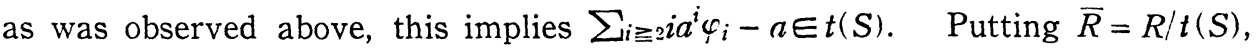
we denote for $x \in R$ the residue class of $x$ modulo $t(S)$ by $\bar{x}$. We have thus

$$
\sum_{i \geqq 2} i \bar{a}^{i} \varphi_{i}-\bar{a}=0 .
$$

Now we assume that $F$ is generated by its elements $\alpha_{1}, \ldots, \alpha_{r}$ and subring $K$ satisfying the condition in our theorem. Consider the elements $\overline{1} \alpha_{1}$, $\ldots, \overline{1} \alpha_{r}$ of the subring $\overline{1} F$ of $\bar{R}$, where $\overline{1}$ denotes the unit element of the field $\bar{R}$; we do not assume the existence of a unit element in $R$ though. They and $\overline{1} K$ together generate $\overline{1} F$, where $\overline{1} K$ is either 0 or an algebraically closed field. If there exists a valuation $\rho$ of the field $\bar{R}$ constant on $\overline{1} K$ such that

$$
\rho(\bar{a})>0, \rho\left(\overline{1} \alpha_{\nu}\right) \geqslant 0(\nu=1, \ldots, r),
$$

then $\rho\left(\overline{1} \varphi_{i}\right) \geq 0, \rho\left(\bar{a}^{i} \varphi_{i}\right) \geqslant i \rho(\bar{a})$ and the above relation will lead to a contra- 
diction $\rho(i \vec{a})=\left(\sum_{1} \geqq 2 i a^{i} \varphi_{i}\right) \geq 2 \rho(\ddot{a})>\rho(\bar{a})$.

We contend that except for the case when $\overline{1} K=0$ and $\bar{R}$ is of characteristic $p \neq 0$ and absolutely algebraic, we may choose $a(\in R$ but $\notin Z)$ so that the existence of such valuation $\rho$ is secured. Indeed, in case $\overline{1} K=0$, this is clear from [9], Hilfssatz 1 ; apply the Hilfssatz to any subfield of $R$ which is a proper simple extension of the subfield $\bar{Z}=Z / t(S)$ on remembering the footnote 1$)$ there. If $\overline{1} K \neq 0$, on the other hand, $\bar{R}$ is transcendental over $\overline{1} K$, because of the assumption $R \neq Z$, which implies $\bar{R} \neq \bar{Z} \supseteqq$ (in fact $\supset$ ) $\overline{1} K$, and an (easy) part of our proof to Hilfssatz 1 in [9] suffices to show the existence of a (nontrivial) valuation $\rho$ of $\bar{R}$ constant on $\overline{1} K$ and satisfying $\rho\left(\overline{1} \alpha_{\nu}\right) \geq 0, \quad(\nu=1$, $\ldots, r), \rho(a-z)>0$ with some $z \in Z$. Denoting $a-z$ by $a$ anew, we have our contension.

In the exceptional case, where thus ( $\overline{1} K=0$ and) $\bar{R}$ is of characteristic $p \neq 0$ and absolutely algebraic, there exists for each element $x$ of $R$ a natural number $m(x)$ such that $\bar{x}-\bar{x}^{m(x)+1}=0$, i.e. $x-x^{m(x)+1} \in t(S)$. Since $t(S) \leqq Z$, we have $x-x^{m(x)+1} \in Z$. Then $R=Z$ by Herstein [4] (cf. also Jacobson [7], $X$, $\$ 2$, Theorem 2).

So, in any case our assumption $R \neq Z$ has led to a contradiction, proving $R=Z$.

The case of subdirectly irreducible $R$, either semisimple or non-semisimple, being thus settled, the transition to the general case, of our theorem, is rather habitual.

What we have done in the above, after the reduction by Drazin's lemma, is indeed to prove.

Proposition. Let $F$ be a commutative ring ( $\exists 1$ ) as in our Theorem. Let $R$ be an algebra (footnote 1$)$ ) over $F$ such that for every element a of $R$ there is a polynomial $b$ in a over $F$ with $a-a^{2} b \in Z$, the center of $R$. Then $R=Z$, i.e. $R$ is commutative.

It is possible, on amplifying the above proof with somewhat complicated considerations in the last part of our proof in [9] (p.26 in particular), further to extend this generalization of Herstein's [5] theorem so as to cover [9], Satz 2 too, but that would be too much to be done here. 


\section{REFERENCES}

[1] R. Arens-I. Kaplansky, Topological representation of algebras, Trans. Amer. Math. Soc. 63 (1948), 457-481.

[2] M. P. Drazin, Algebraic and diagonable rings, Canad. J. Math. 8 (1956), 341-354.

[3] M. P. Drazin, Rings with nil commutator ideals, Rend. Circ. Math. Palermo II ser. 6 (1957), 51-64.

[4] I. N. Herstein, A generalization of a theorem of Jacobson, III, Amer. J. Math. 75 (1953), 105-111.

[5] I. N. Herstein, The structure of a certain class of rings, Amer. J. Math. 75 (1953), 864-871.

[6] N. Jacobson, Structure theory for algebraic algebras of bounded degree, Ann. Math. 46 (1945), 695-707.

[ 7 ] N. Jacobson, Structure of Rings, Amer. Math. Soc. Colloquium Publ. 37 (1956).

[8] M. Nagata-T. Nakayama-T. Tsuzuku, On an existence lemma in valuation theory, Math. Nagoya J. 6 (1953), 59-61.

[9] T. Nakayama, Über die Kommutativität gewisser Ringe, Abh. Math. Sem. Hamburg $20(195 \overline{5}), 20-27$.

[10] Y. Utsumi, On s-rings, Proc. Japan Acad. 33 (1957), 63-66.

\section{Mathematical Institute}

Nagoya University 